\title{
AUMENTO DA AERAÇÃO NASAL APÓS REMOÇÃO DE HÁBITOS DE SUCÇÃO E TERAPIA MIOFUNCIONAL
}

\author{
Nasal Breathing after removing sucking habit \\ and myofunctional therapy
}

Viviane Veroni Degan ${ }^{(1)}$, Regina Maria Puppin-Rontani ${ }^{(2)}$

\begin{abstract}
RESUMO
Objetivo: avaliar os efeitos da associação entre a remoção de hábitos de sucção e a Terapia Miofuncional Orofacial na ampliação da aeração nasal. Métodos: vinte crianças na faixa de etária de quatro anos a quatro anos e oito meses foram distribuídas em dois grupos denominados de: Grupo REM (submetido apenas à remoção de hábitos) e Grupo TMF (submetido à remoção de hábitos e posteriormente à Terapia Miofuncional Orofacial). O fluxo de ar expirado pelas narinas foi registrado por meio do espelho nasal milimetrado de Altmann e quantificado em papel milimetrado denominado de Bloco de Referência do Espelho de Altmann. Os exames foram realizados em três momentos (pré-tratamento, 60 e 180 dias após). Os resultados foram submetidos à análise estatística, utilizando-se Test t para duas amostras independentes e para dados pareados, considerando nível de significância valores $p<0,05$. Resultados: pode-se observar que as médias obtidas da quantificação da aeração nasal após os tratamentos foram significativamente maiores no Grupo TMF que no Grupo REM ( $p<0,0001)$. Conclusão: houve aumento da aeração nasal e conseqüentemente melhora da função respiratória nasal, utilizando a Terapia Miofuncional Orofacial após remoção de hábitos de sucção em crianças na faixa etária de quatro anos.
\end{abstract}

DESCRITORES: Hábitos; Terapia Miofuncional; Sucção; Respiração Bucal

\section{INTRODUÇÃO}

Atualmente vários estudos apontam para alta prevalência de hábitos de sucção nutritivos e não nutritivos e que comumente são utilizados por crianças de maneira prolongada ${ }^{1,2}$ e desta forma são considerados nocivos ao desenvolvimento.

O uso prolongado dos hábitos encontra-se altamente correlacionado a etiologia de más-oclusões, principalmente a mordida aberta anterior ${ }^{2-6} \mathrm{e}$ alterações de estruturas e funções do sistema estomatognático, bem como mastigação, deglutição, respiração e fonoarticulação ${ }^{6-13}$.

A respiração nasal permite que os lábios mantenham-se em contato e a mandíbula em repouso, possibilitando que a língua se aloje dentro da cavidade oral, ficando em contato com o palato, equilibrando-

(1) Fonoaudióloga; Docente do Curso de Pós-graduação em Odontologia do Centro Universitário Herminio Ometto / UNIARARAS; Doutora em Odontologia - Área de Fisiologia Oral pela Faculdade de Odontologia de Piracicaba da Universidade Estadual de Campinas.

(2) Dentista; Professora Titular da Área de Odontopediatria da Faculdade de Odontologia de Piracicaba da Universidade Estadual de Campinas. se com as forças restritivas dos músculos bucinadores, sendo coadjuvante do crescimento transversal da maxila ${ }^{12,14}$.

A respiração predominantemente oral pode estar relacionada a hábitos de sucção prolongados ${ }^{12,15,16}$.

A criança que apresenta este tipo de comportamento em relação aos hábitos pode manter-se com os lábios afastados que se tornam flácidos, deixando de exercer a função de vedamento e, como conseqüência, facilitam o padrão de respiração oral viciosa ${ }^{11,12,14}$. Este padrão respiratório pode determinar modificações no posicionamento de dentes, mandíbula e língua, além de ausência de vedamento da cavidade oral, provocado pela falta de contato dos lábios, determinando respostas neuromusculares inadequadas para o correto crescimento e desenvolvimento craniofacial ${ }^{12,17}$.

A remoção de hábitos de sucção em crianças em fase de dentição decídua pode proporcionar correção ou atenuação de má oclusão do tipo mordida aberta anterior, adequação de estruturas e funções do sistema estomatognático ${ }^{11,18}$. Porém, somente a remoção dos hábitos pode não promover total adequação destas funções, sendo assim, a utilização da Terapia Miofuncional Orofacial pode favorecer o aumento da 
força muscular, provocar mudanças positivas nos padrões funcionais, e assim prevenir desvios no desenvolvimento craniofacial, pois gera adequação de postura de estruturas em repouso e durante a realização de funções do sistema estomatognático ${ }^{11,18-20}$.

Desta forma, o objetivo deste estudo foi avaliar os efeitos da associação entre a remoção de hábitos de sucção e de Terapia Miofuncional Orofacial na ampliação da aeração nasal.

\section{MÉTODOS}

Todos os responsáveis pelos voluntários leram e assinaram um Termo de consentimento livre e esclarecido elaborado de acordo com as diretrizes e normas regulamentadas de pesquisa envolvendo seres humanos atende à Resolução nำ196/96.

A amostra consistiu de 20 pacientes, de ambos os gêneros, segundo os critérios de inclusão como: presença de hábitos de sucção de chupeta e mamadeira, faixa etária de quatro anos a cinco anos incompletos e presença de mordida aberta anterior. Foram assumidos como critérios de exclusão: crianças com outras interferências oclusais, como mordida cruzada anterior e/ou posterior, respiradores orais obstrutivos e presença de algum outro tipo de hábito de sucção que não fosse chupeta e mamadeira. Foram eliminadas da amostra as crianças que não abandonaram os hábitos de sucção de chupeta e mamadeira ao completarem 5 anos de idade e/ou que tiveram os incisivos centrais decíduos esfoliados durante o período do experimento. Todas as crianças apresentavam hábitos de sucção de chupeta, utilizandoa livremente tanto durante o dia quanto à noite, e mamadeira em uma freqüência média de duas vezes ao dia. A idade para remoção dos hábitos citados foi determinada por estudo anterior ${ }^{21}$, cuja amostra era formada por crianças entre as idades de quatro e seis anos.

A amostra foi dividida em dois grupos, aleatoriamente de acordo com o tratamento empregado em:

1- GRUPO REM - com dez crianças que foram submetidas apenas à remoção de hábitos de sucção de chupeta e mamadeira pelo Método de Esclarecimento Modificado ${ }^{22}$.

2- GRUPO TMF - com dez crianças que foram submetidas à remoção de hábitos de sucção de chupeta e mamadeira pelo Método de Esclarecimento Modificado, associado Terapia Miofuncional Orofacial.

Após a entrevista inicial, as crianças foram encaminhadas para a realização dos exames iniciais: exame clínico fonoaudiológico, odontológico, exame radiográfico e documentação fotográfica.

\section{Entrevista inicial:}

A entrevista inicial foi realizada individualmente com cada responsável pela criança, quando foram coletados dados referentes aos hábitos de sucção apresentados naquele momento como freqüência, duração, intensidade, a atitude da criança frente aos hábitos de sucção em locais públicos, comportamento dos pais ou responsáveis em relação aos hábitos, atitudes tomadas em relação à utilização dos hábitos e tentativas anteriores para removê-los. Também foram coletados dados quanto ao comportamento social: freqüência de choro, relacionamento com outras crianças, e com os pais. Ainda, os pais foram questionados quanto à alimentação da criança, substituição de alimentos por leite, histórico de má-oclusão familiar, saúde geral, padrão respiratório, doenças já apresentadas, desenvolvimento motor, preferências da criança com relação aos jogos e situações agradáveis. Estes dados foram coletados para que se montasse um programa individual de remoção dos hábitos de sucção, com base no Método de Esclarecimento Modificado ${ }^{22}$.

\section{Tratamento Miofuncional Orofacial:}

Logo após a remoção dos hábitos apresentados, as crianças do grupo TMF foram submetidas a 8 sessões de Terapia Miofuncional Orofacial realizadas uma vez por semana durante 30 minutos. Durante estas sessões a criança realizava os exercícios isométricos e funcionais e recebia um calendário ${ }^{11}$ para que os executasse também em casa uma vez ao dia. Os calendários eram trocados semanalmente, durante as consultas. Um adulto, responsável pela criança, participava do final da sessão para que também aprendesse a executar os exercícios corretamente e pudesse, assim, supervisionar a criança.

A Terapia Miofuncional Orofacial foi realizada visando:

- Aumento da força dos músculos: orbicular da boca superior, orbicular da boca inferior, bucinadores e músculos linguais. Estes músculos foram trabalhados por meio de exercícios isométricos.

- Adequação de estruturas durante o repouso: adequação do posicionamento dos lábios (em vedamento) e adequação do posicionamento lingual (em região de papila retro-incisal).

- Adequação das seguintes funções do sistema estomatognático: deglutição e respiração (nasal). Foram utilizados exercícios para promover as funções adequadamente.

- Foram utilizados exercícios preparatórios de deglutição de líquidos e alimentos sólidos.

- Para favorecer a utilização da respiração nasal usou-se como estímulos os processos de inspiração expiração. O fluxo de ar proveniente da expiração foi anotado em bloco de referência do espelho de Altmann. O uso da respiração nasal foi associado ao processo de automatização de lábios vedados e língua em região de papila retroincisal em repouso. 
- A respiração foi avaliada por meio de prova utilizando-se Espelho nasal milimetrado de Altmann. Este era colocado sob as narinas da criança enquanto ela mantinha inspiração e expiração sem instrução prévia. O fluxo de ar expirado pelas narinas foi quantificado a partir do registro obtido por meio do espelho que era reproduzido em papel igualmente milimetrado denominado de bloco de referência. Os espaços marcados pelo fluxo de ar eram contados para atribuição de valor em centímetro quadrado $\left(\mathrm{cm}^{2}\right)$.

Para cada voluntário foi traçado um plano de tratamento personalizado que visava à adequação das estruturas e funções citadas.

A remoção de hábitos de sucção de chupeta e mamadeira foi efetuada antes da realização da Terapia Miofuncional Orofacial.

A força muscular foi analisada em artigo anteriormente publicado ${ }^{11}$.

Análise dos resultados

Os resultados foram analisados por meio de exames clínicos e fotográficos, realizados previamente e aos 60 e 180 dias após a remoção dos hábitos de sucção.

Para o Grupo REM, os hábitos foram removidos e a partir da data de remoção esperou-se 60 dias e 180 dias para a realização dos exames.

Para o Grupo TMF, os hábitos foram removidos e as oito sessões semanais de Terapia Miofuncional Orofacial foram realizadas, totalizando-se dois meses após a remoção dos hábitos, realizando-se então, a segunda avaliação, e após 180 dias a terceira. Portanto, todas as crianças da amostra foram submetidas concomitantemente à realização dos exames iniciais (pré-tratamento), 60 e 180 dias após a remoção dos hábitos de sucção.

\section{Exame Clínico:}

As crianças participantes foram submetidas à avaliação miofuncional orofacial, por uma fonoaudióloga avaliadora que não tinha conhecimento sobre em qual grupo a criança estava inserida (Kappa 85\%), constituindo um experimento do tipo duplo cego.

Foram avaliadas as seguintes estruturas: lábios, mento, língua, bochechas, oclusão e funções como deglutição, fala e respiração.

A respiração foi avaliada por meio de prova utilizando-se Espelho nasal milimetrado de Altmann. Este era colocado sob as narinas da criança enquanto ela mantinha respiração sem instrução prévia. O fluxo de ar expirado pelas narinas produzia uma marca no espelho que era reproduzida em papel igualmente milimetrado denominado de "bloco de referência". Os espaços marcados pelo fluxo de ar foram então contados para atribuição de um valor.

O projeto de pesquisa teve a aprovação do Comitê de Ética em Pesquisa da Faculdade de Odontologia FOP/UNICAMP sob n 19/2000.
Os dados coletados foram submetidos à análise estatística, utilizando-se Teste t para duas amostras independentes e o Teste t para dados pareados, considerando nível de significância valores $p<0,05$.

\section{RESULTADOS}

A função respiração foi quantificada $\mathrm{em}^{\mathrm{cm}}{ }^{2}$ por meio do espelho de Altmann, sendo que os resultados das avaliações encontram-se na Tabela 1.

Pode-se observar que as médias obtidas pela quantificação da aeração nasal após os tratamentos, no Grupo TMF foram significativamente maiores que no Grupo REM ( $p<0,0001)$, ressaltando o maior aproveitamento da aeração nasal para as crianças que receberam a associação dos tratamentos. Esta constatação pode ser observada na segunda e terceira avaliações não diferindo estatisticamente entre si em ambos os grupos estudados, REM ( $p=0,7905)$ e TMF ( $p=0,9352)$. Entretanto, diferiram significativamente da avaliação inicial, em ambos os grupos REM ( $p=0)$ e TMF ( $p=0,0024)$.

Ainda na Tabela 1 pode-se observar que na avaliação inicial os grupos não diferiram entre si, apresentando menor utilização do espaço aéreo nasal $(p=0,0912)$. Na segunda avaliação o Grupo TMF apresentou aumento significativo da utilização do espaço aéreo nasal $(p=0,0062)$, mantendo em média na terceira avaliação $(p=0,0095)$ aumento significativo.

A Figura 1 ilustra as médias das áreas medidas no espelho de Altmann obtidas durante o período do estudo para a amostra.

Observa-se que os grupos REM e TMF iniciaram com graus similares de aeração nasal. Na segunda avaliação observou-se melhores padrões respiratórios para ambos os grupos, porém com o grupo TMF exibindo melhores resultados. Este perfil foi mantido até a terceira avaliação.

Tabela 1 - Distribuição da área $\left(\mathrm{cm}^{2}\right)$ da quantificação da aeração nasal pelas crianças nos períodos de avaliação

\begin{tabular}{lccc}
\hline & & Avaliações \\
& Inicial & 60 dias & 180 dias \\
\hline REM & $10,70 \mathrm{Aa}$ & $18,10 \mathrm{Ab}$ & $18,40 \mathrm{Ab}$ \\
TMF & $14,10 \mathrm{Aa}$ & $26,40 \mathrm{Bb}$ & $26,50 \mathrm{Bb}$ \\
\hline
\end{tabular}

REM - Grupo submetido à remoção de hábitos

TMF - Grupo submetido à remoção de hábitos e posteriormente à terapia miofuncional orofacial

Percentuais seguidos por mesma letra Maiúscula na comparação entre grupos não diferem entre si pelo teste t para 2 amostras independentes $(p>0,05)$

Percentuais seguidos por mesma letra Minúscula na comparação dentro do mesmo grupo não diferem entre si pelo teste t para dados pareados $(p>0,05)$ 


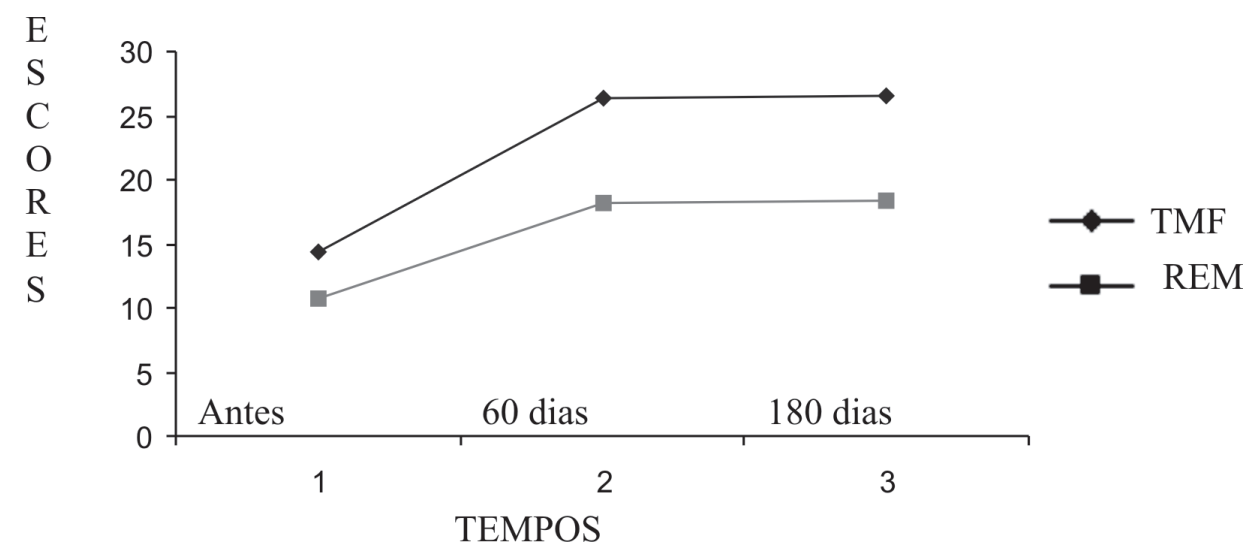

REM - Grupo submetido à remoçao de hábitos

TMF - Grupo submetido à remoção de hábitos e posteriormente à terapia miofuncional orofacial

\section{Figura 1 - llustração gráfica dos escores médios exibidos pelos grupos tratados durante o período de estudo quanto à aeração nasal}

\section{DISCUSSÃO}

Constatou-se que a TMF produziu aumento significativo na aeração nasal analisada por meio do uso do espelho nasal milimetrado de Altmann.

A quantificação da aeração nasal mostrou que houve aumento desta, decorrente da remoção de hábitos de sucção aos 60 dias, mantendo-se até o final do experimento, porém no grupo que foi submetido à remoção dos hábitos associada à Terapia Miofuncional, as alterações positivas foram mais evidentes e também permaneceram até o último exame. Estes fatos confirmam a efetividade da Terapia Miofuncional no restabelecimento da função respiratória nasal, corroborando afirmações de outros autores ${ }^{23}$ e achados de outro estudo ${ }^{24}$ que utilizaram a Terapia Miofuncional no restabelecimento da função respiratória nasal, e encontraram valores iniciais de $14,82 \mathrm{~cm}^{2}$, finalizando o experimento com valores de $26,33 \mathrm{~cm}^{2}$, sendo estes próximos aos valores iniciais e finais encontrados para o Grupo TMF $\left(14,10 \mathrm{~cm}^{2}\right.$ inicial e $26,40 \mathrm{~cm}^{2}$ final - Tabela 1).

O aumento da força dos músculos orbiculares da boca proporcionada pela Terapia Miofuncional ${ }^{11}$ foi um fator importante, pois auxiliou na correta postura de lábios em contato, mantendo-os vedados em repouso, sendo a maior garantia de que a respiração seria realizada corretamente pelo nariz ${ }^{11,25}$.

No grupo que foi submetido somente a remoção de hábitos de sucção também houve aumento da aeração nasal que mostrou ser indicativo de que hábitos de sucção prolongados favorecem a respiração oral devido à manutenção de padrão de ausência de vedamento labial ${ }^{14}$.

O tempo de utilização da Terapia Miofuncional Orofacial, neste estudo foi de 8 sessões, tempo também utilizado por outro estudo ${ }^{26}$ no qual os autores utilizaram a Intervenção Fonoaudiológica Breve e obtiveram melhora para a respiração diurna e a postura correta dos lábios em repouso habitual.

Várias mães relataram que as crianças, após os tratamentos, passaram a dormir com lábios vedados e houve diminuição ou mesmo ausência de episódios de roncos e escape de saliva durante o sono, apresentando melhor qualidade deste, novamente corroborando as afirmações de outro estudo ${ }^{24}$.

A respiração nasal pode ser considerada imprescindível para o bom desenvolvimento de estruturas e funções orofaciais e conseqüentemente, o adequado crescimento e desenvolvimento craniofacial ${ }^{12,27,28}$, portanto deve ser restabelecida precocemente, evitando-se assim alterações posturais e no sistema estomatognático ${ }^{13,17}$.

Com esse propósito a intervenção precoce promovida pela remoção de hábitos de sucção associada à Terapia Miofuncional pode favorecer padrões de crescimento e desenvolvimento craniofacial apropriados, reabilitando funções e estruturas do sistema estomatognático ${ }^{11,18,29}$.

\section{CONCLUSÃO}

Por meio deste estudo foi possível verificar que houve aumento da aeração nasal, adequação do vedamento labial e aumento da resistência dos lábios e conseqüentemente melhora da função respiratória nasal utilizando a Terapia Miofuncional Orofacial após remoção de hábitos de sucção em crianças na faixa etária de quatro anos a cinco anos incompletos.

\section{AGRADECIMENTOS}

À FAPESP pela bolsa de doutorado (processo 99/ 12166-1), à Secretaria de Saúde do Município de Piracicaba - SP e à Fonoaudióloga ROSEMARY 


\section{ABSTRACT}

Purpose: to evaluate the influence of removing pacifier and baby bottle sucking habit and Myofunctional Therapy in increasing nasal airing. Methods: twenty children aged up to four year, were distributed in two groups according to the treatment, and named: REM Group (only submitted to habits removal) and FMT Group (submitted to habits removal and later to Myofunctional Therapy). The data obtained from the air flow expired by the nostrils were obtained by using the millimeter nasal mirror of Altmann and quantified in millimeter paper (reference block). The exams were accomplished in three times (pretreatment, 60 and 180 days after treatment). The data were submitted to t Test for two independent samples and the paired t Test $(p<0.05)$. Results: it was observed that the increase of the nasal airing after treatments were significantly superior for the FMT Group than for the REM Group $(p<0.0001)$. Conclusion: Myofunctional Therapy was able to improve increase of nasal airing and consequently enhancement of nasal breathing.

KEYWORDS: Habits; Myofunctional Therapy; Suction; Mouth Breathing

\section{LEITE MACIEL ORTIZ pela colaboração. REFERÊNCIAS}

1.Degan VV, Puppin-Rontani RM. Prevalence of pacifier-sucking habits and successful methods to eliminate them: a preliminary study. J Dent Child. 2004; 71(2):148-51.

2.Katz CR, Rosenblatt A, Gondim PP. Nonnutritive sucking habits in brazilian children: effects on deciduous dentition and relationship with facial morphology. Am J Orthod Dentofacial Orthop. 2004; 126(1):53-7.

3.Warren JJ, Bishara SE, Steinbock KL, Yonezu T, Nowak AJ. Effects of oral habits' duration on dental characteristics in the primary dentition. J Am Dent Assoc. 2001; 132(12):1685-93.

4. Charchut SW, Allred EN, Needleman HL. The effects of infant feeding patterns on the occlusion of the primary dentition. J Dent Child. 2003; 70(3):197-203. 5. Emmerich A, Fonseca L, Elias AM, Medeiros UV. The relationship between oral habits, oronasopharyngeal alterations, and malocclusion in preschool children in Vitoria, Espirito Santo, Brazil. Cad Saude Publica. 2004; 20(3):689-97.

6. Bertoldi P, Felício M, Matsumoto MA. Effect of the early intervention of oral habits on the development of dental occlusion. Pró-Fono R Atual Cient. 2005; 17(1):37-44.

7.Garreto AL. Orofacial myofunctional disorders related to malocclusion. Int J Orofacial Myology. 2001; 27:44-54.

8. Martinez MI, Assêncio-Ferreira VJ. Hábito orais viciosos versus alterações de oclusão dentária: prevalência de crianças com oclusão normal e hábitos orais viciosos. Rev CEFAC. 2001; 3(2):127-31. 9.Zardetto CG, Rodrigues CR, Stefani FM. Effects of different pacifiers on the primary dentition and oral myofunctional strutures of preschool children. Pediatr
Dent. 2002; 24(6):552-60.

10. Felício CM, Ferreira-Jeronymo RR, Ferriolli BHVM, Freitas RLRG. Análise da associação entre sucção, condições miofuncionais orais e fala. Pró-Fono R Atual Cient. 2003; 15(1):31-40.

11. Degan VV, Puppin-Rontani RM. Terapia miofuncional e hábitos orais infantis. Rev CEFAC. 2004; 6(4):396-404.

12. Andrade FV, Andrade DV, Araújo AS, Ribeiro ACCR, Deccax LDG, Nemr K. Alterações estruturais de órgãos fonoarticulatórios e más oclusões dentárias em respiradores orais de 6 a 10 anos. Rev CEFAC. 2005; 7(3):318-25.

13. Bicalho GP, Motta ARM, Vicente LCC. Avaliação da deglutição em crianças respiradoras orais. Rev CEFAC. 2006; 8(1):50-5.

14. Felício CM. Fonoaudiologia aplicada a casos odontológicos: motricidade oral e audiologia. São Paulo: Pancast; 1999. 243 p.

15. Chevitarese AB, Della Valle D, Moreira TC. Prevalence of malocclusion in 4-6 year old brazilian children. J Clin Pediatr Dent. 2002; 27(1):81-5.

16. Trawitzki LVV, Anselmo-Lima WT, Melchior MO, Grechi TH, Valera FC. Breast-feeding and deleterious oral habits in mouth and nose breathers. Rev Bras Otorrinolaringol. 2005; 71(6):747-51.

17. Lessa FCR, Enoki C, Feres MFN, Valera FCP, Lima WTA, Matsumoto MAN. Influência do padrão respiratório na morfologia craniofacial. Rev Bras Otorrinolaringol. 2005; 71(2):156-60.

18. Degan VV, Puppin-Rontani RM. Remoção de hábitos e terapia miofuncional: restabelecimento da deglutição e repouso lingual. Pró-Fono R Atual Cient. 2005; 17(3):375-82.

19.Pascal HHM, Van Lieshout PH, Bose A, Namasivayam AK. Physiological effects of an 8-week mechanically aided resistance facial exercise program. Int J Orofacial Myology. 2002; 28:49-73. 
20. Jefferson Y. Orthodontic diagnosis in young children: beyond dental malocclusions. Gen Dent. 2003; 51(2):104-11.

21. Degan VV, Boni RC, Almeida RC. Idade adequada para remoção de chupeta e/ou mamadeira, na faixa etária de 4 a 6 anos. J Orthop-Orthod Pediatr Dent. 2001; 3:5-16.

22. Boni RC, Almeida RC, Degan VV. Utilização do método de esclarecimento para remoção do hábito de sucção de chupeta e/ou mamadeira. J OrthopOrthod Pediatr Dent. 2001; 2(11):16.

23. Schievano D, Puppin-Rontani RM, Bérzin F. Influence of myofunctional therapy on the perioral muscles: clinical and electromyographic evaluations. J Oral Reabil. 1999; 26:564-9.

24. Araújo VC, Bitar ML. Efeito da terapia miofuncional oral em crianças respiradoras bucais segundo enfoque da promoção de saúde. Disponível em: URL: <http://www.respiremelhor.com.br/emarb/3emarb/ resumo14.htm> Acesso: 06 out. 2003.

25. Camargo MCF, Azevedo JO, Briso MLG. Dispositivo indutor de vedamento labial: divel. J Bras Ortodon Ortop Facial. 2001; 6(6):256-62.

26. Bacha SMC, Ríspoli CM. Myofunctional therapy: brief intervention. Int J Orofacial Myology. 1999; 25:3747.

27. Ribeiro F, Bianconi CC, Mesquita MCM, AssêncioFerreira VJ. Respiração oral: alterações oclusais e hábitos orais. Rev CEFAC. 2002; 4(3):187-90.

28. Yamaguchi H, Sueishi K. Malocclusion associated with abnormal posture. Bull Tokyo Dent Coll. 2003; 44(2):43-54.

29. Haruki T, Morita H, Kanomi R, Kawabata J. Oral morphology and tongue habits. Int J Orofacial Myology. 1995; $21: 4-8$.

RECEBIDO EM: 13/10/06

ACEITO EM: 21/02/07

Endereço para correspondência:

Rua Abertina Simões Oliveira, 238

Limeira - SP

CEP: $13484-260$

Tel: (19) 34419274 / 34516649 / 91998545

Fax: (19) 34424817

E-mail:vvdegan@yahoo.com.br ; viviane@degan.com.br

Rev CEFAC, São Paulo, v.9, n.1, 55-60, jan-mar, 2007 